\title{
Micobacteriosis identificadas en el Laboratorio Nacional de Referencia de Colombia entre 2012 y 2016
}

\author{
Claudia Llerena, Angélica Valbuena, Angie Paola Zabaleta \\ Grupo de Micobacterias, Laboratorio Nacional de Referencia, \\ Instituto Nacional de Salud, Bogotá, D.C., Colombia
}

\begin{abstract}
Introducción. En los últimos años se ha observado un aumento en la prevalencia de micobacteriosis ocasionadas por micobacterias no tuberculosas, las cuales hoy se consideran como agentes patógenos emergentes. Su presencia depende de varios factores, como los antecedentes clínicos, el estado de salud de la persona afectada y la presencia de estos microorganismos en el agua, el suelo y los animales, entre otros.

Objetivo. Describir las micobacteriosis identificadas en el Laboratorio Nacional de Referencia de Micobacterias del Instituto Nacional de Salud, entre 2012 y 2016.

Materiales y métodos. Se hizo un análisis retrospectivo que incluyó 273 pacientes con micobacteriosis. Las variables analizadas fueron el tipo de micobacteriosis, el agente etiológico y los factores de riesgo asociados.

Resultados. El 57,1\% de los casos presentó micobacteriosis pulmonar, el $26 \%$, cutánea, el 10,6 \%, diseminada, y el 2,6\%, linfática. La comparación entre la forma pulmonar y la extrapulmonar evidenció que el complejo Mycobacterium avium se presenta con mayor frecuencia en las micobacteriosis pulmonares, en tanto que $M$. abscessus fue más frecuente en las extrapulmonares. Los pacientes con micobacteriosis pulmonar presentaban antecedentes de tuberculosis con mayor frecuencia que aquellos con las formas extrapulmonares.

Conclusión. Estos hallazgos resaltan la importancia del diagnóstico diferencial entre las especies del complejo $M$. tuberculosis y las micobacterias no tuberculosas, ya que estas últimas son resistentes genéticamente a los fármacos convencionales contra la tuberculosis.
\end{abstract}

Palabras clave: Mycobacterium; micobacterias no tuberculosas; factores de riesgo; coinfección; infecciones oportunistas; Colombia.

doi: https://doi.org/10.7705/biomedica.v38i0.4143

Mycobacterioses identified in the National Reference Laboratory of Colombia from 2012 to 2016

Introduction: In recent years, there has been an increase in the prevalence of mycobacterioses caused by non-tuberculous mycobacteria, which are considered as emerging pathogens. Their presence depends on several factors such as the clinical history, the health status of the affected person, and the presence of these microorganisms in the water, the soil, and the animals, among others.

Objective: To describe the mycobacteria and the etiological agent identified in isolates received at the Laboratorio Nacional de Referencia de Micobacterias of the Instituto Nacional de Salud between 2012 and 2016.

Materials and methods: We conducted a retrospective analysis of samples from 273 patients with mycobacterioses. We analyzed the following variables: mycobacteriosis type, etiological agent, and associated predisposing factors.

Results: $57.1 \%$ of the cases presented pulmonary mycobacteriosis; $26 \%$, cutaneous; $10.6 \%$, disseminated, and $2.6 \%$, lymphatic. We found the Mycobacterium avium complex more frequently in pulmonary mycobacteriosis, while $M$. abscessus was more frequent in the extrapulmonary types of the disease. Patients with pulmonary mycobacteriosis had a history of tuberculosis more frequently than those with extrapulmonary forms.

Conclusion: These findings highlight the importance of the differential diagnosis between $M$. tuberculosis complex species and non-tuberculous mycobacteria since the latter are genetically resistant to conventional antituberculosis drugs.

Key words: Mycobacterium; non-tuberculous mycobacteria; risk factors; coinfection; opportunistic infections; Colombia.

doi: https://doi.org/10.7705/biomedica.v38i0.4143

\section{Contribución de los autores:}

Todas las autoras participaron en la realización de los ensayos de laboratorio, la depuración y el análisis de la información, y en la escritura del documento. 
En los últimos años se ha observado un aumento en la prevalencia de micobacteriosis ocasionadas por micobacterias no tuberculosas, las cuales se consideran agentes patógenos emergentes. Su presencia depende de los antecedentes clínicos y el estado de salud de la persona afectada, por ejemplo, inmunosupresión debida al virus de la inmunodeficiencia humana (HIV), alteraciones estructurales del pulmón, como las bronquiectasias, la fibrosis quística y la enfermedad pulmonar obstructiva crónica (EPOC), las cuales favorecen la colonización de las vías respiratorias con estos gérmenes. A estos se suman hoy otros factores, como la gran variedad de entornos geográficos en los que se encuentran estos agentes patógenos y su relación con el medio ambiente, ya que tienen una amplia distribución en el agua, el aire, los suelos y los animales. En muchas ocasiones, los cuadros clínicos que producen se confunden con la tuberculosis, lo que favorece el avance de la enfermedad y el deterioro clínico, el cual puede llevar a la muerte cuando el tratamiento no es el adecuado (1-4).

En muchos de los estudios llevados a cabo a nivel mundial, se reconoce que la micobacteriosis con mayor prevalencia es la pulmonar, y los agentes etiológicos más frecuentes han sido Mycobacterium avium intracellulare, $M$. chelonae, $M$. fortuitum, $M$. abscessus, $M$. marinum, $M$. xenopi y $M$. kansasii $(1,3,5,6)$.

En Colombia, el sistema de vigilancia en salud pública no exige el informe de las micobacterias no tuberculosas, lo cual dificulta establecer con qué frecuencia se presentan. En los trabajos a nivel nacional que documentan su presencia como agentes patógenos, se ha evidenciado un incremento en el número de casos, especialmente en personas infectadas con el HIV, o en quienes tienen enfermedad pulmonar o son sometidos a procedimientos cosmetológicos. Las especies identificadas son $M$. chelonae, $M$. fortuitum, $M$. avium intracellulare, $M$. abscessus, $M$. gordonae, M. scrofulaceum y M. avium, entre otras (4,7-11).

\footnotetext{
Correspondencia:

Angélica Valbuena, Grupo de Micobacterias, Laboratorio Nacional de Referencia, Instituto Nacional de Salud, Avenida calle $26 \mathrm{~N}^{\circ}$ 51-20, Bogotá, D.C., Colombia

Teléfono: (571) 2207700 , extensión 1260

yvalbuena@ins.gov.co

Recibido: 16/11/17; aceptado: 26/05/18
}

En este contexto, el objetivo de nuestro estudio fue describir los casos de micobacteriosis diagnosticados en el Laboratorio Nacional de Referencia de Micobacterias del Instituto Nacional de Salud entre el 2012 y el 2016.

\section{Materiales y métodos}

Se hizo un análisis retrospectivo de la base de datos del laboratorio que incluía a 273 pacientes en cuyos aislamientos se habían identificado micobacterias no tuberculosas. Para determinar la especie, se utilizaron los estuches comerciales Genotype $\mathrm{CM}^{\mathrm{TM}}$, que permite la identificación de las especies más comunes, y Genotype AS ${ }^{\mathrm{TM}}$ para aquellos casos en los que la prueba de Genotype $\mathrm{CM}^{\mathrm{TM}}$ no reconoció la especie micobacteriana. La técnica se basa en la extracción, la amplificación con iniciadores marcados con biotina y la hibridación inversa, proceso que se hizo siguiendo las indicaciones del fabricante $(12,13)$.

Como controles internos, se utilizaron las cepas ATCC de $M$. tuberculosis $\left(25618^{\mathrm{TM}}\right), M$. avium $\left(15769^{\mathrm{TM}}\right)$ y $M$. fortuitum $\left(6841^{\mathrm{TM}}\right)$, así como los controles de extracción y amplificación establecidos por el Laboratorio Nacional de Referencia.

La fuente de información fue el formato único de vigilancia de las micobacterias, recibido con cada uno de los casos. Las variables analizadas fueron edad, sexo, casos detectados por año, procedencia geográfica, tipo de micobacteriosis (pulmonar, cutánea, diseminada o linfática), agente etiológico identificado y factores predisponentes, entre los cuales se consideraron el tratamiento antituberculoso previo, la infección simultánea con el HIV (en aquellos casos en que se había registrado el resultado de esta prueba), la EPOC, la diabetes, la desnutrición y otros factores inmunosupresores no definidos. Los casos que no disponían de esta información se registraron como sin factor predisponente asociado.

Para el análisis estadístico, se utilizó la prueba z entre las variables de agente etiológico identificado y factor predisponente asociado para las formas pulmonar y extrapulmonar, y el porcentaje de casos recibidos a lo largo de los años de estudio. El área crítica de significación se estableció como p menor de 0,05 , con un intervalo de confianza del $95 \%$.

\section{Resultados}

Entre 2012 y 2016, se diagnosticaron 273 casos de micobacteriosis en el Laboratorio Nacional de Referencia: 41 (15\%) casos en el 2012, 28 $(10,3 \%)$ en el 2013, 55 (20,1\%) en el 2014, 69 
(25,3 \%) en el 2015 y 80 (29,3\%) en el 2016. La comparación del número de casos recibido a lo largo de este periodo, tomando como referencia el 2012 , evidenció un aumento significativo a partir de $2015(p<0,05)$.

Los casos provenían de 23 entidades territoriales. Las que aportaron el mayor número fueron: Bogotá, con 64 (23,4\%), Atlántico, con 48 (17,6\%), Santander, con 40 (14,7\%), Antioquia, con 18 (6,6\%), y Bolívar con, 13 (4,8\%), casos en los que se encontró el $67 \%$ de las micobacteriosis estudiadas (cuadro 1).

El $57,1 \%$ de los casos correspondió a micobacteriosis pulmonar, el $26 \%$, a cutánea, el 10,6 \%, a diseminada, el 2,6\%, a linfadenitis, y en el 3,7\% de los casos no se tenía la información para establecer el sistema comprometido (cuadro 2).

El principal agente causante de la micobacteriosis pulmonar fue el complejo $M$. avium en 81 casos $(51,9 \%)$. Entre los factores predisponentes asociados con esta micobacteriosis, se encontró el tratamiento previo para tuberculosis en $52(33,3 \%)$ casos; el $81 \%$ de los pacientes era mayor de 40 años, con una mediana de edad de 57 , y el $60 \%$ correspondió al sexo masculino.

Mycobacterium abscessus fue la principal causa de enfermedad cutánea, con 39 casos (54,9\%), siendo la inmunosupresión el factor más frecuentemente asociado (7\%); el 76,1\% de estos pacientes tenía entre 21 y 50 años de edad, con una mediana de 34 ; el 78,9 \% correspondió al sexo femenino.

El agente etiológico más frecuente en los casos de micobacteriosis diseminada fue el complejo $M$, avium con 20 casos; el $51 \%$ de los pacientes con micobacteriosis diseminada era positivo para el HIV. El 82,7 \% de estos pacientes tenía entre 21 y 60 años, con una mediana de 43; el 79,3\% correspondió al sexo masculino.

Solo se detectaron $7(2,6 \%)$ casos de linfadenitis por micobacteriosis, $5(71,4 \%)$ de ellos secundarios a infección por el complejo M. avium. Seis $(85,7 \%)$ fueron seropositivos para el HIV. Las edades de los pacientes oscilaban entre 4 y 39 años y el $71,4 \%$ correspondió al sexo masculino.

En 5 de los 10 casos en que no había suficiente información para establecer la región anatómica afectada, se identificó el complejo $M$. avium, y el factor predisponente asociado más frecuentemente registrado fue el antecedente de tratamiento antituberculoso, en 4 casos. Las edades oscilaron entre 21 y 70 años y el 7 correspondió al sexo femenino.
Cuadro 1. Número y porcentaje de casos enviados al Laboratorio Nacional de Referencia del Instituto Nacional de Salud por las entidades territoriales, Colombia, 2012-2016

\begin{tabular}{lcc}
\hline Entidad territorial & \multicolumn{2}{c}{$\begin{array}{c}\text { Casos enviados al } \\
\text { Instituto Nacional de Salud } \\
\mathbf{n}(\%)\end{array}$} \\
\hline Bogotá & 64 & $(23,4)$ \\
Atlántico & 48 & $(17,6)$ \\
Santander & 40 & $(14,7)$ \\
Antioquia & 18 & $(6,6)$ \\
Bolívar & 13 & $(4,8)$ \\
Valle del Cauca & 10 & $(3,7)$ \\
Norte de Santander & 10 & $(3,7)$ \\
Magdalena & 8 & $(2,9)$ \\
Quindío & 8 & $(2,9)$ \\
Cesar & 8 & $(2,9)$ \\
Risaralda & 7 & $(2,6)$ \\
Cundinamarca & 6 & $(2,2)$ \\
Córdoba & 5 & $(1,8)$ \\
Meta & 5 & $(1,8)$ \\
Nariño & 4 & $(1,5)$ \\
Cauca & 4 & $(1,5)$ \\
La Guajira & 4 & $(1,5)$ \\
Casanare & 3 & $(1,1)$ \\
Huila & 3 & $(1,1)$ \\
Caldas & 2 & $(0,7)$ \\
Tolima & 1 & $(0,4)$ \\
San Andrés & 1 & $(0,4)$ \\
Sucre & 1 & $(0,4)$ \\
Total & 273 & $(100)$ \\
\hline
\end{tabular}

Al comparar las formas pulmonares y las extrapulmonares (cutánea, diseminada, linfática), se estableció que el complejo $M$. avium fue más el frecuente en la micobacteriosis pulmonar, en tanto que $M$. abscessus y $M$. chelonae lo fueron en las extrapulmonares $(p<0,05)$ (cuadro 3 ).

El factor predisponente más frecuente en las formas pulmonares $(p<0,05)$ fue el tratamiento previo para la tuberculosis (cuadro 3 ).

\section{Discusión}

Durante los cinco años analizados, se produjo un aumento de los casos confirmados como micobacteriosis y se observó una diferencia estadísticamente significativa a partir del 2015. Ello podría deberse a que, desde el 2014, el Laboratorio Nacional de Referencia utiliza para la identificación de la especie una técnica molecular más sensible y específica, la cual permite una mejor caracterización de las especies si se compara con las pruebas convencionales usadas en años anteriores.

Por otra parte, en la red de laboratorios se han implementado nuevos métodos, como la inmunocromatografía, y técnicas basadas en la amplificación de $A D N$, lo cual permite saber de forma 
Cuadro 2. Tipo de micobacteriosis, agente etiológico identificado, factor predisponente, edad y sexo de los pacientes, Colombia, 2012-2016

\begin{tabular}{|c|c|}
\hline Micobacteriosis pulmonar $(n=156)$ & n (\%) \\
\hline \multicolumn{2}{|l|}{ Especie identificada } \\
\hline Complejo Mycobacterium avium & $81(51,9)$ \\
\hline M. abscessus & $32(20,5)$ \\
\hline M. fortuitum & $15(9,6)$ \\
\hline M. kansasii & $6(3,8)$ \\
\hline M. chelonae & $5(3,2)$ \\
\hline M. mucogenicum & $4(2,6)$ \\
\hline M. szulgai & $4(2,6)$ \\
\hline Mycobacterium spp. & $4(2,6)$ \\
\hline M. simiae & $3(1,9)$ \\
\hline M. gordonae & $1(0,6)$ \\
\hline M. scrofulaceum & $1(0,6)$ \\
\hline \multicolumn{2}{|l|}{ Factor predisponente asociado } \\
\hline Personas con tratamiento previo para tuberculosis & $52(33,3)$ \\
\hline Personas con infección simultánea con HIV & $31(19,9)$ \\
\hline EPOC & $10(6,4)$ \\
\hline Otros factores inmunosupresores & $7 \quad(4,5)$ \\
\hline Diabetes & $6(3,8)$ \\
\hline Desnutrición & $1(0,6)$ \\
\hline Sin factor predisponente asociado & $49(31,4)$ \\
\hline Micobacteriosis cutánea $(n=71)$ & n (\%) \\
\hline \multicolumn{2}{|l|}{ Especie identificada } \\
\hline M. abscessus & $39(54,9)$ \\
\hline M. fortuitum & $15(21,1)$ \\
\hline M. chelonae & $10(14,1)$ \\
\hline Complejo M. avium & $4(5,6)$ \\
\hline M. marinum & $2(2,8)$ \\
\hline M. kansasii & $1(1,4)$ \\
\hline \multicolumn{2}{|l|}{ Factor predisponente asociado } \\
\hline Otros factores inmunosupresores & $5(7,0)$ \\
\hline Diabetes & $1(1,4)$ \\
\hline Persona con infección simultánea por HIV & $3(4,2)$ \\
\hline Sin factor predisponente asociado & $62(87,3)$ \\
\hline Micobacteriosis diseminada $(n=29)$ & n (\%) \\
\hline \multicolumn{2}{|l|}{ Especie identificada } \\
\hline Complejo M. avium & $20(69,0)$ \\
\hline M. fortuitum & $3(10,3)$ \\
\hline M. simiae & $2(6,9)$ \\
\hline M. kansasii & $2(6,9)$ \\
\hline M. abscessus & $1(3,4)$ \\
\hline M. malmoense & $1(3,4)$ \\
\hline \multicolumn{2}{|l|}{ Factor predisponente asociado } \\
\hline Persona que vive con HIV & $15(51,7)$ \\
\hline Otros factores inmunosupresores & $5(17,2)$ \\
\hline Personas con tratamiento previo para tuberculosis & $1(3,4)$ \\
\hline Diabetes & $1(3,4)$ \\
\hline Sin factor predisponente asociado & $7(24,1)$ \\
\hline Micobacteriosis linfática $(n=7)$ & n (\%) \\
\hline \multicolumn{2}{|l|}{ Especie identificada } \\
\hline Complejo M. avium & $5(71,4)$ \\
\hline M. fortuitum & $1(14,3)$ \\
\hline M. szulgai & $1(14,3)$ \\
\hline \multicolumn{2}{|l|}{ Factor predisponente asociado } \\
\hline Persona con infección simultánea por HIV & $6(85,7)$ \\
\hline Sin factor predisponente asociado & $1(14,3)$ \\
\hline Sin información sobre tipo de micobacteriosis $(n=10)$ & n (\%) \\
\hline \multicolumn{2}{|l|}{ Especie identificada } \\
\hline Complejo M. avium & $5(50)$ \\
\hline M. abscessus & $3(30)$ \\
\hline M. fortuitum & $2(20)$ \\
\hline \multicolumn{2}{|l|}{ Factor predisponente asociado } \\
\hline Personas con tratamiento previo para tuberculosis & $4(40)$ \\
\hline Diabetes & $1(10)$ \\
\hline Sin factor predisponente asociado & $5(50)$ \\
\hline
\end{tabular}

EPOC: enfermedad pulmonar obstructiva crónica rápida si un aislamiento pertenece o no al complejo M. tuberculosis, así como una mayor remisión al Laboratorio Nacional de Referencia de aquellos cultivos en que se sospecha la presencia de micobacterias no tuberculosas. Además, desde el 2009 los lineamientos nacionales establecen el cultivo como método de diagnóstico en personas con sospecha de micobacteriosis $(14,15)$.

El mayor número de casos se registró en Bogotá y en los departamentos de Atlántico y Santander, donde las redes de prestación de servicios de salud tienen más acceso al cultivo como método de diagnóstico.

En la mayoría de los estudios realizados en el país, se ha relacionado la presencia de micobacterias no tuberculosas con la presencia del HIV y con los procedimientos estéticos. No se registran estudios en los que se haya comparado el comportamiento de estas micobacterias como agentes patógenos causantes de la enfermedad pulmonar y de las formas extrapulmonares (4,7-10). En el 2017, Llerena, et al., documentaron que las micobacterias causantes de enfermedad pulmonar entre el 2012 y el 2014 eran del complejo M. aviar (62,7\%) y $M$. abscessus (30,3\%).

El presente análisis incluyó información de dos años más y se evidenció que la tendencia fue similar. Sin embargo, en este estudio se identificaron como agentes etiológicos $M$. simiae y $M$. mucogenicum, los cuales no se habían descrito antes (11).

En el estado de São Paulo en Brasil, Ueki, et al., determinaron que en $48 \%$ de los 1.248 casos con micobacteriosis, esta era de tipo pulmonar, principalmente debida al complejo $M$. avium, con $51 \%$, dato similar a lo encontrado en nuestro estudio. Estos autores identificaron $M$. kansasii como el segundo agente más frecuente. En el presente análisis, se reconoció a $M$. abscessus como la segunda especie más frecuente, con $20,5 \%$ de los casos. En los artículos consultados se reporta que la presencia de micobacterias no tuberculosas varía según la localización geográfica de los casos (16).

En Estados Unidos, Prevots, et al., describieron las especies causantes de micobacteriosis pulmonar entre el 2004 y el 2006, y lo mismo hicieron Simons, et al., en un análisis de 25 artículos publicados en diferentes países del este de Asia. En los dos estudios se reportaron las bacterias del complejo M. avium como los principales agentes involucrados, seguidas de $M$. abscessus, lo cual coincide 
Cuadro 3. Comparación de micobacteriosis pulmonar y extrapulmonar, Colombia, 2012 -2016

\begin{tabular}{|c|c|c|c|c|c|}
\hline \multirow[t]{3}{*}{ Variable } & \multicolumn{5}{|c|}{ Tipo de micobacteriosis } \\
\hline & \multicolumn{2}{|c|}{ Pulmonar $(n=156)$} & \multicolumn{2}{|c|}{ Extrapulmonar $(n=107)$} & \multirow[t]{2}{*}{$\mathbf{p}$} \\
\hline & $\mathbf{n}$ & $\%$ & $\mathrm{n}$ & $\%$ & \\
\hline \multicolumn{6}{|l|}{ Especie identificada } \\
\hline Complejo M. avium & 81 & 51,9 & 29 & 27,1 & $<0,05$ \\
\hline M. abscessus & 32 & 20,5 & 40 & 37,4 & $<0,05$ \\
\hline M. fortuitum & 15 & 9,6 & 19 & 17,8 & 0,808 \\
\hline M. chelonae & 5 & 3,2 & 10 & 9,3 & $<0,05$ \\
\hline M. kansasii & 6 & 3,9 & 3 & 2,8 & 0,568 \\
\hline M. mucogenicum & 4 & 2,6 & 0 & 0 & 0,247 \\
\hline M. szulgai & 4 & 2,6 & 1 & 0,9 & 0,623 \\
\hline M. simiae & 3 & 1,9 & 2 & 1,9 & 0,668 \\
\hline M. gordonae & 1 & 0,6 & 0 & 0 & 0,849 \\
\hline M. marinum & 0 & 0 & 2 & 1,9 & 0,321 \\
\hline M. malmoense & 0 & 0 & 1 & 0,9 & 0,849 \\
\hline M. scrofulaceum & 1 & 0,6 & 0 & 0 & 0,849 \\
\hline Mycobacterium spp. & 4 & 2,6 & 0 & 0 & 0,247 \\
\hline \multicolumn{6}{|l|}{ Factor predisponente asociado } \\
\hline Personas con tratamiento previo para tuberculosis & 52 & 33,3 & 1 & 0,9 & $<0,05$ \\
\hline Persona con infección simultánea por HIV & 31 & 19,9 & 24 & 22,4 & 0,728 \\
\hline EPOC & 10 & 6,4 & 0 & 0 & --- \\
\hline Otros factores inmunosupresores & 7 & 4,5 & 10 & 9,4 & 0,187 \\
\hline Diabetes & 6 & 3,9 & 2 & 1,9 & 0,581 \\
\hline Desnutrición & 1 & 0,6 & 0 & 0 & 0,849 \\
\hline Sin datos & 49 & 31,4 & 70 & 65,4 & $<0,05$ \\
\hline
\end{tabular}

EPOC: enfermedad pulmonar obstructiva crónica

con los datos de este estudio y es relevante si se tiene en cuenta que todos estos análisis corresponden a regiones geográficas diferentes $(5,6,17)$.

En el presente estudio, se comparó la micobacteriosis pulmonar con las extrapulmonares para establecer las diferencias en cuanto a la presencia de los factores predisponentes asociados, y se comprobó que el antecedente de tratamiento antituberculoso fue uno de los más relevantes en las formas pulmonares, lo cual podría deberse a que la primera vez que los pacientes recibieron el tratamiento solo se les hizo una baciloscopia y no se hicieron cultivos en las muestras, por lo cual no se comprobó si se trataba de una micobacteria no tuberculosa; además, la tuberculosis pulmonar deja bronquiectasias como secuela y estas pueden ser colonizadas por dichas micobacterias. De ahí la importancia de utilizar pruebas para identificar las especies en todos los casos sospechosos de micobacteriosis con este antecedente, ya que las micobacterias no tuberculosas tienen la capacidad de colonizar el pulmón cuando se presentan daños estructurales como los producidos por el complejo $M$. tuberculosis, en especial cuando se hace un diagnóstico tardío $(1,3,6,7)$.

Se encontró que la micobacteriosis pulmonar era más frecuente en personas mayores de 51 años, principalmente en los hombres mayores de 60 . En el estudio de Prevots, et al., se evidenció que la prevalencia de la micobacteriosis aumentaba siete veces en hombres y mujeres mayores de 60 años, lo cual coincide con los datos del presente estudio (17-19).

La micobacteriosis cutánea se asocia con traumas, heridas quirúrgicas o inyecciones y, especialmente, con procedimientos estéticos en los cuales usualmente se emplean sustancias sin control sanitario o no se cumplen los protocolos de desinfección, higiene y bioseguridad. Esta situación se ha descrito en varios países de Suramérica y afecta en un mayor porcentaje a las mujeres $(8,9,19)$.

En un estudio realizado entre el 2004 y el 2007 por García, et al., se analizaron 80 casos de problemas en la piel asociados con procedimientos estéticos y, en $33(41,2 \%)$ de ellos, se identificó alguna micobacteria no tuberculosa como causante de la infección: 57,5 \%, M. chelonae, 24,2 \%, $M$. fortuitum, y 18,2 \%, M. abscessus. En Venezuela se evaluaron 49 pacientes que desarrollaron infección en la piel y los tejidos blandos después de una mesoterapia y, en $81,6 \%$ de los casos, se identificó una micobacteria: $M$. abscessus en el $55 \%$ de ellos, $M$. fortuitum en el $25 \%$ y $M$. chelonae en el $10 \%$, datos similares a los de este estudio. Por otra parte, se evidenciaron algunas diferencias porcentuales con el estudio de García, 
las cuales pueden estar relacionadas con el uso de pruebas de identificación fenotípica que dificultan la diferenciación de especies estrechamente relacionadas, como $M$. abscessus y $M$. chelonae, las cuales pueden identificarse con mayor precisión al emplear métodos moleculares (19-23).

La micobacteriosis diseminada y la linfática se deben generalmente al complejo $M$. avium y se asocian con inmunosupresión. Los especímenes ingresan principalmente por la vía gastrointestinal y se ha demostrado que algunos linajes del complejo son más virulentos y favorecen la invasión de las células HT-29, lo cual permite la diseminación a múltiples órganos, especialmente en personas inmunocomprometidas. En este estudio, la forma diseminada y la linfática presentaban inmunosupresión en $68,9 \%$ y $85,7 \%$ de los casos, respectivamente, lo cual coincide con lo reportado en la literatura mundial (24).

En algunos estudios se han identificado mutaciones en genes implicados en la producción y el control de la interleucina 12 y el interferón gamma, así como en el control de la reacción celular y el desarrollo hematopoyético, lo que hace a los individuos más propensos a padecer micobacteriosis diseminada (25).

En la revisión de Osorio, et al., para caracterizar las complicaciones infecciosas pulmonares en pacientes con HIV tras 20 años del inicio de la terapia antirretroviral combinada, se encontró que las especies de mayor relevancia clínica fueron el complejo $M$. avium, $M$. kansasii, $M$. fortuitum, $M$. abscessus, $M$. malmoense y $M$. simiae, lo cual coincide con lo hallado en el presente estudio. Se ha establecido que los recuentos de CD4 menores de 50 células/ $\mu$ y las cargas virales mayores de 100.000 copias $/ \mathrm{ml}$ aumentan el riesgo de presentar micobacteriosis $(17,26,27)$.

Ueki, et al., describieron en su trabajo que el $97,8 \%$ de los casos de micobacteriosis diseminada se debía al complejo $M$. avium; en los pacientes con infección concomitante con el HIV, esta especie también predominó (80,2 \%) (16).

En los últimos años, el diagnóstico de las micobacterias no tuberculosas como agentes etiológicos se ha incrementado con el desarrollo y la introducción de métodos más sensibles en la rutina de los laboratorios, así como con el reconocimiento de nuevas especies asociadas con enfermedades (28-31). En Colombia, Castro, et al., identificaron las siguientes especies de micobacterias utilizando la técnica de análisis de patrones de restricción: $M$. fortuitum, $M$. abscessus, $M$. intracellulare, $M$. gordonae, M. avium, M. chelonae, M. scrofulaceum, M. szulgai, M. terrae y M. malmoense; al comparar estos datos con los nuestros, se observó que el uso de la metodología Genotype CM/ASTM ha permitido detectar especies como $M$. kansasii, $M$. mucogenicum y $M$. simiae $(10,14)$.

Una limitación de este estudio la constituyó el diligenciamiento del formato único de vigilancia de las micobacterias, ya que la información no es completa en cuanto a la presencia de otros factores predisponentes asociados, así que, en los casos en que no se registró la información completa, no fue posible asegurar que estos no existieran, lo cual constituye un sesgo.

En todos los estudios citados, se resalta la importancia del diagnóstico diferencial entre especies del complejo $M$. tuberculosis y las micobacterias no tuberculosas. Asimismo, es importante que los médicos hagan la correlación con los criterios clínicos, radiológicos y bacteriológicos establecidos por la American Thoracic Society (ATS), mediante los cuales es posible hacer el diagnóstico adecuado y, en consecuencia, prescribir el tratamiento idóneo en estos casos $(1,32)$.

El reto de los servicios de salud es fortalecer la capacidad de los médicos tratantes para sospechar, diagnosticar y tratar los casos de micobacterias no tuberculosas, pues cada vez se describen más especies asociadas con enfermedades. Es fundamental que las fuentes de información proporcionen datos que permitan correlacionar los factores predisponentes asociados ya descritos y documentar otros nuevos, incluidos el entorno ambiental de los pacientes, las actividades laborales y las intervenciones quirúrgicas, entre otros.

\section{Agradecimientos}

A los profesionales y a los integrantes de los equipos de apoyo técnico y administrativo de las secretarías de salud del país, así como a los del Laboratorio de Micobacterias del Instituto Nacional de Salud.

\section{Conflicto de intereses}

Las autoras declaramos que no existe ningún tipo de conflicto de intereses.

\section{Financiación}

Este trabajo se hizo con recursos del contrato 757/ 13 suscrito entre el Departamento Administrativo 
de Ciencia y Tecnología, Colciencias, y la Dirección de Redes en Salud Pública del Instituto Nacional de Salud.

\section{Referencias}

1. Farga V, Caminero J. Tuberculosis. Tercera edición. Santiago de Chile: Editorial Mediterráneo; 2011. p. 449-67.

2. Piersimoni C, Scarparo C. Pulmonary infections associated with non-tuberculous mycobateria in inmmunocompetent patients. Lancent Infect Dis. 2008;8:323-34. https://doi. org/10.1016/S1473-3099(08)70100-2

3. Katoch V. Infections due to non-tuberculous mycobacteria (NTM). Indian J Med Res. 2004;120:290-304.

4. Murcia M, Gómez J, Alvarado F, Bustillo J, Deivels E, Gómez B, et al. Prevalence of mycobacterial in HIV positive patients in Bogotá. Rev Colomb Neumol. 2001;13:249-61. https://doi.org/10.1186/1471-2334-1-21

5. Prevots R, Marras T. Epidemiology of human pulmonary infection with non-tuberculous mycobacteria: A review. Clin Chest Med. 2015;36:13-34. https://doi.org/10.1016/j. ccm.2014.10.002

6. Simons $\mathbf{S}$, van Ingen $\mathbf{J}$, Hsueh $\mathbf{P R}$, van Hung $\mathbf{N}$, Dekhuijzen PN, Boeree MJ, et al. Nontuberculous mycobacteria in respiratory tract infections, eastern Asia. Emerg Infect Dis. 2011;17:343-9. https://doi.org/10.3201/ eid170310060

7. Montúfar F, Madrid C, Montufar M, Aguilar C, Saldarriaga C, Mesa M, et al. Caracterización de pacientes hospitalizados con infecciones causadas por micobacterias no tuberculosas en un hospital de alta complejidad en Colombia. Infectio. 2014;18:135-42. https://doi.org/10.1016/j. infect.2014.06.002

8. Garzón M, Orjuela D, Naranjo O, Llerena C. Micobacterias no tuberculosas en Colombia, 1995-2003. Inf Quinc Epidemiol Nac. 2005;10:161-76.

9. García L, Garzón M, Orjuela D, Mejía G, Llerena C. Micobacterias no tuberculosas asociadas a procedimientos de mesoterapia en Colombia, 2004-2007. Infectio. 2010; 14:93-6. https//doi:10.1016/S0123-9392(10)70096-5

10. Castro C, Puerto G, García L, Orjuela D, Llerena C, Garzón M, et al. Identificación molecular de micobacterias no tuberculosas mediante el análisis de los patrones de restricción, Colombia 1995-2005. Biomédica. 2007;27:43946. https://doi.org/10.7705/biomedica.v27i3.206

11. Llerena C, Valbuena Y, Zabaleta A, Gómez T. Enfermedad pulmonar causada por complejo Mycobacterium avium y M. abscessus. Acta Médica Colombiana. 2017;42:26-9.

12. Hain Lifescience. Genotype Mycobacterium $\mathrm{CM}^{\mathrm{TM}}$. Versión 1.0. Hardwiesenstraße, Nehren: Hain Lifescience; 2011.

13. Hain Lifescience. Genotype Mycobacterium AS ${ }^{\mathrm{TM}}$. Versión 1.0. Hardwiesenstraße, Nehren: Hain Lifescience; 2010.

14. Russo C, Tortoli E, Menichella D. Evaluation of the new genotype Mycobacterium assay for identification of mycobacterial species. J Clin Microbiol. 2006;44:1769-75. https://doi.org/10.1128/JCM.44.2.334-339.2006

15. Dirección General de Salud Pública, Ministerio de la Protección Social. Circular externa 058 de 2009. Lineamientos para el manejo programático de tuberculosis y lepra en Colombia. Bogotá: Ministerio de la Protección Social; 2009. Fecha de consulta: 15 de febrero de 2018. Disponible en: https://www.minsalud.gov.co/sites/rid/Lists/ BibliotecaDigital/RIDE/DE/DIJ/circular-externa-0007de-2015.pdf.

16. Ueki S, Martins $\mathbf{M}$, Telles $\mathbf{M}$, Virgilio M, Giampaglia $\mathbf{C}$, Chimara E, et al. Nontuberculous mycobacteria: Species diversity in Sao Paulo state, Brazil. J Bras Patol Med Lab. 2005;41:1-8. https://doi.org/10.1590/S1676-244420050001 00003

17. Prevots D, Shaw P, Strickland D, Jackson L, Raebel M, Blosky M. Nontuberculous mycobacterial lung disease prevalence at four integrated health care delivery systems. Am J Respir Crit Care Med. 2010;182:970-6. https://doi. org $/ 10.1164 / \mathrm{rccm} .201002-03100 \mathrm{C}$

18. Valdivies J, Sánchez R, Fernández S, Osés L. Síndrome de Lady Windermere como causa de tos crónica. A propósito de un caso. An Fac Med. 2015;76:285-7. https:// doi.org/10.15381/anales.v76i3.11241

19. Rivera O, Guevara A, Escalona A, Oliver M, Pérez A, Piquero J, et al. Infecciones en tejidos blandos por micobacterias no tuberculosas secundarias a mesoterapia. ¿Cuánto vale la belleza? Enferm Infecc Microbiol Clin. 2006;24:302-6. https://doi.org/10.1157/13089664

20. Sanudo A, Vallejo F, Sierra M, Hoyos J, Yepes S, Wolff $\mathbf{J}$, et al. Nontuberculous mycobacteria infection after mesotherapy: Preliminary report of 15 cases. Int $\mathrm{J}$ Dermatol. 2007;46:649-53. https://doi.org/10.1111/j.13654632.2007.02976.x

21. Munayco C, Grijalva C, Culqui D, Bolarte J, SuárezOgnio L, Quispe N, et al. Outbreak of persistent cutaneous abscesses due to Mycobacterium chelonae after mesotherapy sessions, Lima, Perú. Rev Saúde Pública. 2008;42:146-9. https://doi.org/10.1590/S0034-891020080 00100020

22. García X, Barnadas M, Dalmau J, Coll P, Gurgui, Alomar A. Mycobacterium abscessus infection secondary to mesotherapy. Clin Exp Dermatol. 2008;33:658-9. https://doi. org/10.1111/j.1365-2230.2008.02869.x

23. Carbonne A, Brossier F, Arnaud I, Bougmiza I, Caumes $\mathrm{E}$, Meningaud $\mathrm{J}$, et al. Outbreak of nontuberculous mycobacterial subcutaneous infections related to multiple mesotherapy injections. J Clin Microbiol. 2009;47:1961-4. https://doi.org/10.1128/JCM.00196-09

24. Wu U, Holland S. Host susceptibility to non-tuberculous mycobacterial infections. Lancet Infect Dis. 2015;8:968-80. https://doi.org/10.1016/S1473-3099(15)00089-4

25. Bustamante J, Boisson-Dupuisa S, Abel L, Casanova J. Mendelian susceptibility to mycobacterial disease: Genetic, immunological, and clinical features of inborn errors of IFN-y immunity. Semin Immunol. 2014;26:454-70. https:// doi.org/10.1016/j.smim.2014.09.008

26. Osorio J, Álvarez D, Barreto J, Casanova M, Vargas H, Giraldo G, et al. Infecciones pulmonares en pacientes con VIH 20 años después de la terapia antiretroviral combinada. ¿Qué ha cambiado? Infectio. 2015;20:180-9. https// doi.org/10.1016/j.infect.2015.08.002

27. Barnes A, Rojo S, Moretto H. Prevalencia de micobacteriosis y de tuberculosis en pacientes de un hospital de referencia de la provincia de Córdoba. Rev Argent Microbiol. 2004;36:170-3. 
28. Telenti A, Marchesi F, Bals M, Bally F, Bodmer T. Rapid identification of Mycobacteria to the species level by polymerase chain reaction and restriction enzyme analysis. J Clin Microbiol. 1993;31:175-8.

29. Tobler N, Pfunder M, Herzog K, Frey J, Altwegg M. Rapid detection and species identification of Mycobacterium spp. using real-time PCR and DNA-Microarray. J Microbiol Methods. 2006;66:116-24. https//doi.org/10.1016/j.mimet. 2005.10.016

30. Ng E, Wong M, Poon T. Advances in MALDI mass spectrometry in clinical diagnostic applications. Top Curr Chem. 2014;336:139-75. https://doi.org/10.1007/1282012413
31. Alcaide F, Palop-Borrás B, Domingo D, Tudó G. Aplicación de la espectrometría de masas en micobacterias. Enferm Infecc Microbiol Clin. 2016;34(Supl.2):31-5. https:// doi.org/10.1016/S0213-005X(16)30188-4

32. Griffith D, Aksamit T, Brown-Elliott B, Catanzaro A, Daley C, Gordin F, et al. ATS Mycobacterial Diseases Subcommittee; American Thoracic Society; Infectious Disease Society of America. An official ATS/IDSA statement: Diagnosis, treatment, and prevention of non-tuberculous mycobacterial diseases. Am J Respir Crit Care Med. 2007; 175:367-416. https://doi.org/10.1164/rccm.200604-571ST 